\title{
LHomme
}

LHOMME Rever fancease danthropologie

$217 \mid 2016$

Varia

\section{L'alliance matrimoniale des sociétés traditionnelles entre discours épidictique et discours apodictique, entre dette symbolique et échange}

The Matrimonial Alliance in Traditional Societies: Between Epideictic and

Apodictic Discourses, Between Symbolic Debt and Exchange

\section{Charles-Henry Pradelles de Latour}

\section{OpenEdition \\ Journals}

Édition électronique

URL : http://journals.openedition.org/lhomme/28836

DOI : $10.4000 /$ /homme.28836

ISSN : 1953-8103

Éditeur

Éditions de l'EHESS

\section{Édition imprimée}

Date de publication : 25 février 2016

Pagination : 45-59

ISSN : 0439-4216

\section{Référence électronique}

Charles-Henry Pradelles de Latour, «L'alliance matrimoniale des sociétés traditionnelles entre discours épidictique et discours apodictique, entre dette symbolique et échange ", L'Homme [En ligne], 217 | 2016, mis en ligne le 24 février 2018, consulté le 01 mai 2019. URL : http://

journals.openedition.org/lhomme/28836 ; DOI : 10.4000/lhomme.28836 


\title{
L'alliance matrimoniale des sociétés traditionnelles entre discours épidictique et discours apodictique, entre dette symbolique et échange
}

\author{
Charles-Henry Pradelles de Latour
}

\begin{abstract}
L' AnAlyse de l'alliance matrimoniale, objet de cet article, a été largement induite par l'expérience que j'ai vécue lors de mon premier terrain africain dans une chefferie bamiléké, à savoir la chefferie Bangoua. Pour situer les lieux, précisons que les Bamiléké, résident au Cameroun, dans la province du Centre-Ouest, sur des plateaux de mille mètres d'altitude où ils bénéficient de conditions climatiques et géographiques exceptionnelles ayant favorisé leur développement démographique. Ce pays très peuplé, dont la densité démographique s'élève par endroits à plus de 200 habitants au $\mathrm{km}^{2}$, a été divisé au milieu du XIXe siècle en une centaine de chefferies d'inégale importance, dirigées par un chef supérieur et un conseil de neuf notables. La chefferie Bangoua, située dans le département du Ndé, comprend 5500 habitants. La densité de la population est telle que, la terre étant rare, beaucoup d'hommes ont dû pour survivre et s'enrichir s'adonner au commerce en sus de leurs occupations agraires. C'est pourquoi les Bamiléké sont connus au Cameroun pour être des commerçants entreprenants, hiérarchiquement divisés en rangs de prestige et de richesse - notables, fils de chef, serviteurs, villageois -, et ayant su évoluer et s'adapter à la modernité tout en restant fidèles à leurs traditions.
\end{abstract}

\section{Une nomination en tant qu'allié du chef}

Dès mon arrivée, j'ai été chaleureusement reçu par fô Watong, le chef bangoua, qui m'a proposé de résider avec ma famille dans son palais traditionnel. Grâce à cet accueil, j'ai pu me mettre rapidement au travail. Un vieux catéchiste ayant renié la religion chrétienne m’apprenait la langue le matin et, l'après-midi, aidé par un jeune traducteur, j'ai commencé 
à mener diverses enquêtes auprès des habitants. Tout s'engageait bien, lorsqu'à l'instigation d'un serviteur malveillant le chef accusa publiquement le secrétaire de mairie de vouloir l'"empoisonner », c'est-à-dire de vouloir le tuer par la sorcellerie. Cette accusation de régicide ${ }^{1}$, qui révélait une tension entre les deux pouvoirs en place, le chef traditionnel de la chefferie d'un côté et le pouvoir de l'administration étatique de l'autre, éveilla aussitôt mon intérêt. Une affaire de sorcellerie, n'est-ce pas une belle expérience ethnologique? En réalité, je déchantai car le pays se divisa instantanément en deux factions qui ne se parlaient plus. Ayant noué de bons rapports avec les deux partis, je me retrouvai assis entre deux chaises. Mon hôte, le chef, supportait mal que j'interroge ses opposants et me foudroyait du regard quand il apprenait que j'avais vu le secrétaire de mairie ou un de ses acolytes. Ceux-ci m'expliquaient que leur chef était rétrograde, prisonnier de croyances arriérées, et m'incitaient, au nom de la modernité que je représentais par ma couleur de peau, à les soutenir. De plus, le chef exerçait une certaine pression sur moi en me laissant entendre qu'il m'avait reçu pour que j'étudie les coutumes du pays, donc pour que je les défende. Tout semblait compliqué, dans quelle galère m'étais-je embarqué ? Rester neutre dans une affaire de sorcellerie relevait du casse-tête.

Ce conflit fit rapidement boule de neige, tout le monde en parlait. Le préfet et le sous-préfet du Ndé en débattaient, et les élites bangoua résidant à Yaoundé et à Douala commencèrent à s'alarmer, car il était intolérable que les habitants d'une même chefferie ne forment pas "une seule bouche ", une unité. Un gendre du chef qui remplissait la fonction de secrétaire d'État au ministère des Finances à Yaoundé fit, avec quelques autres personnalités, comprendre à son beau-père qu'il devait renoncer à son accusation, sinon les élites ne lui verseraient plus l'aide financière dont il avait besoin pour assurer son rang de chef supérieur. L'argument "trébuchant" fit mouche car le chef accepta peu après de se réconcilier officiellement avec le secrétaire de mairie lors de la fête bisannuelle des Bangoua, qui a lieu en novembre. Ce jour-là, les deux hommes scellèrent l'alliance du sang au cours d'une réunion privée en présence de notables et de personnes faisant autorité. Je n'y assistai pas mais, après la cérémonie, plusieurs des participants ayant appartenu aux partis antagonistes vinrent nous saluer, mon épouse et moi, en nous remerciant d'être restés neutres dans cette affaire.

1. Les chefs bamiléké, appelés fồ ("chef») dans le langage courant, sont intronisés comme des rois, cf. Charles-Henry Pradelles de Latour (1997: 177-183). 
La vie reprit son cours comme auparavant. Le jour du petit marché qui suivit cet événement, mefô Tchutuo, l'héritière de la mère du chef Nônô ${ }^{2}$ - le père du chef Watong -, se mit à m'appeler "Tiènja' ", nom d'éloge réservé aux fils des filles du chef bangoua, lesquels sont dans cette société patrilinéaire les alliés du chef. Les Tiènja' étant nombreux sur la place du marché, je n'y prêtai guère attention, jusqu'au moment où trois hommes, amusés, me prirent par la main et m'amenèrent devant la reine-mère qui s'adressa à moi en parlant bangoua sous les éclats de rire de ceux qui étaient présents. Selon l'usage local, je lui offris quelques morceaux de noix de cola, et elle me dit: "Merci Tiènja' ". J'étais baptisé. Ce surnom, qui me lia à mon hôte - devenu mon grand-père maternel - par une dette symbolique d'alliance matrimoniale, nous amena à partager une proximité bienveillante sans hostilité, ni intimité. Le chef comprit d'emblée que cette appellation allait changer la relation filiale qu'il voulait entretenir avec moi, mais elle réjouit les habitants du pays qui se sentirent autorisés à m’obliger à parler leur langue et à plaisanter avec moi. J'appris ainsi que les rites que je croyais abandonnés étaient toujours pratiqués. En me levant plus tôt, j'ai pu y assister souvent et retrouver un vif intérêt à mon travail. L'enquête ethnographique qui s'était transformée en chemin de croix devint soudain passionnante.

Cette nomination, qui me conférait un surnom et un statut d'allié par rapport au chef bangoua, a fait acte. Précisons qu'un acte a tout d'abord un caractère inaugural que n'a pas l'action. L'acte instaure un commencement ${ }^{3}$. Tout acte est, d'une certaine façon, un acte de naissance qui signa ici le début de ma carrière d'ethnologue. Ensuite, l'acte crée une irréversibilité. Il prévient tout retour en arrière. L'affaire de sorcellerie qui m'avait tant troublé fut oubliée, on n'en parla plus. Enfin, troisième point, l'acte est rétroactif ${ }^{4}$. Il implique une coupure dont les effets se manifestent dans l'après-coup. Cette rétroactivité m'a poussé dans ma vie professionnelle à revenir à ce point de départ et à élaborer différentes théories visant à rendre compte de la dette symbolique d'alliance matrimoniale, jusqu'à ce que je m'aperçoive qu'elle ne relève d'aucune connaissance, et qu'en cela elle est au principe du désir et de la sexualité qui, fondamentalement, participent d'une énigme.

2. Lorsque le chef Nônô a été intronisé chef, sa mère est devenue mefô ("Reine-mère ») et, à la mort de celle-ci, sa fille puis la fille de sa fille ont hérité de son titre et de ses biens. Il s'agit, ici, de cette dernière.

3. Jacques Lacan, L'Acte psychanalytique, séminaire inédit du 10 janvier 1967.

4. Jacques Lacan, La Logique du fantasme, séminaire inédit du 15 février 1968. 
Cet acte induit par un manque originel est différent des actes traumatiques qui perturbent rétroactivement les victimes leur vie durant, des actes juridiques qui, par leurs sentences positives ou négatives, rappellent la loi, et des actes de parole qui conferent au dire un faire (Austin 1970:41; Ducrot 1972), tels les énoncés dont un locuteur se porte garant en pariant, promettant ou prêtant serment. Ces actes, différents par les positions subjectives qui les sous-tendent, ont pour caractéristique d'être antérieurs au sujet qu'ils déterminent. Celui-ci, agi, est un sujet-effet. En ce sens les actes s'opposent aux actions qui ont pour origine un sujetagent doté d'une intention focalisée sur un objectif à atteindre. Alors que l'acte est conditionné par son point de départ, l'action l'est par son point d'arrivée. Elle peut aussi être réversible et, à cet effet, remanier, différer ou arrêter les moyens mis en œuvre pour atteindre son but. Enfin, en termes de subjectivité, l'acte est fondateur du sujet-effet qui, d'abord, agi de l'extérieur, avalise ensuite la coupure qui l'engendre et les pertes que celle-ci provoque, tandis que l'action se présente comme pouvant être un gain pour le sujet-agent qui se réalise dans son effectuation et se valorise (ou se dévalorise) par son résultat.

En somme, le sujet-agi est interne à l'acte qui le produit, et le sujetagent externe à l'action qu'il conduit. J'ai utilisé cette opposition dans mon dernier livre pour différencier les thérapies de type psychanalytique branchées sur la subjectivité des comportements quotidiens que l'enquête ethnographique décrit en termes d'objectivité (Pradelles de Latour 2014 : 122-132). Puis, après la parution de cet ouvrage, la lecture de Jacques, le sophiste. Lacan, logos et psychanalyse, de Barbara Cassin, m’a révélé que l'action et l'acte découlent respectivement des discours apodictique et épidictique qui se sont opposés à Athènes au Ve siècle avant J.-C. (Cassin 2012 : 91-95). Cet apport euristique m’a amené, du même coup, à mieux distinguer mon interprétation de l'alliance matrimoniale des Bamiléké de celle de Lévi-Strauss. Aussi vais-je reprendre les principaux éléments de ces deux discours afin de montrer comment en procèdent deux conceptions de l'alliance matrimoniale.

\section{Les discours apodictique et épidictique}

Le discours apodictique, qui postule que la vérité est donnée avant la parole, est instauré par Parménide, enseigné par l'École éléatique et appliqué par Platon et Aristote à leur philosophie. Le discours épidictique, qui soutient à l'inverse que la vérité se révèle dans l'après-coup du dire, est l'apanage des sophistes, dont Protagoras et Gorgias sont les figures les plus marquantes. L'enjeu de cette opposition tient initialement au fait que 
Parménide, en initiateur de la connaissance rationnelle déconnectée de la croyance, énonce qu'il n'y a que deux voies: "La première est celle de l'être, impossible qu'il ne soit pas, c'est le chemin de la vérité; la seconde où le non-être est nécessaire, c'est le sentier où l'on ne trouve rien à quoi se fier" (in Parménide 1955: 79). Autrement dit, l'être est vrai, le nonêtre faux, et la vérité est l'être Un, qui est d'un seul tenant à la fois penser et être, c'est-à-dire l'identique (Ibid.). Platon s'appuie sur ce postulat tenu pour universel pour fonder ontologiquement l'unité des idées dont il faut s'attacher à reconnaître, derrière leur apparence, l'être véritable, lequel, stable et immuable, constitue la réalité belle à contempler. Aristote, qui ne reconnaît pas cette transcendance, postule pour sa part que les êtres sont uns dans leurs fins que recèlent leurs essences, substances et formes. Poussant plus loin que son prédécesseur le réalisme du discours apodictique, il ancre l'être non plus dans l'idée en soi, mais dans les espèces dont les essences définissent les propriétés et régissent le devenir en puissance. "La nature ", dit-il, " ne fait rien sans dessein " (De caelo, 291 b 13). Pour dissiper toute confusion entre les espèces, il les définit à l'aide d'une dizaine de catégories, et pour déjouer les impasses du raisonnement, il crée la syllogistique qui soumet le dire à la loi de non-contradiction. Ainsi, parler (legein) et signifier (semenainein) s'équivalent (Cassin 1986: 7), l'énoncé étant résolument déconnecté de l'énonciation.

À ce discours fondé sur une vérité apodictique une et première, ou dernière, qui détermine universellement aussi bien le monde des hommes que la nature des choses, les sophistes répondent au contraire que la vérité épidictique est plurielle et seconde, car elle dépend essentiellement des lois que les hommes se donnent par convention dans les cités. La loi est-elle de la nature, comme les philosophes présocratiques ont commencé de l'affirmer, ou celle des hommes, comme les débats des assemblées démocratiques le laissent ostensiblement présager ? Tel est, selon William K. C. Guthrie, le dilemme fondamental - physis ou nomos - auquel les anciens Grecs sont confrontés, et que traduit l'opposition entre philosophes et sophistes (1976:29, 66).

En raison du présupposé nominaliste des sophistes, Protagoras pense que les lois humaines, également justes et injustes, participent d'une double raison, dissos logos (Untersteiner 1993 [1967] : 51), qui, sous des formes différentes, est au principe des controverses politiques et des envolées poétiques. Contre les Éléates, il affirme sans ambages qu' " une chose à la fois bonne et mauvaise, est et n'est pas" (Ibid.: 119). "L'homme étant, pour lui, la mesure de toute chose » (Ibid.: 119 sq.), il incite chacun à trouver sa place dans la cité en se formant et en se défendant à l'aide d'arguments convaincants et de discours appropriés. D'où l'apologie de 
l'enseignement de la vertu et la défense de la rhétorique qu'il soutient contre Socrate 5 . Contrairement à Parménide qui explique que les sensations - froid/chaud, doux/amer - sont fallacieuses, il y a recours pour fonder empiriquement la raison sur les perceptions et montrer que si deux opinions sont également vraies, elles n'ont pas la même validité car elles n'ont pas le même effet: "La vérité est moins un jugement qu'une opération» (Cassin 2012: 76). Protagoras ne cherche pas à définir le vrai et le faux, mais à tirer des situations le meilleur afin de faire passer une personne d'un état à un autre. En définitive, la vérité est pour lui temporaire et particulière, c'est ce dont on peut être persuadé après délibération. Bien que résolument subjectiviste, il ne prône pas pour autant l'anarchie. À l'instar de ses contemporains, il est un adepte de la concorde et de l'égalité ${ }^{6}$, même s'il reconnaît qu'elles ne sont jamais parfaites.

Gorgias, pour sa part, s'érige contre les Éléates en faisant l'apologie du non-être que ceux-ci rejettent dans le domaine du mensonge et de l'irrationalité. Selon un de ses arguments typiquement grecs, si Scylla, la chimère de Sicile que les marins connaissent bien, peut être pensée, c'est qu'un non-étant existe - le non-être est. Cette contradiction étant, selon lui, au fondement même de la mythologie et de la dialectique qui sous-tendent la tragédie. Là, en effet, l'ordre - l'être - s'oppose au chaos le non-être -, et la justice arbitraire des dieux à la justice distributive des hommes dans une lutte effrayante et bouleversante. Selon cette conception littéraire du discours élevée au-dessus des opinions de façon différente de celle de la philosophie, le drame de la raison subséquent au drame des êtres humains est propagé par un artefact du langage, telles l'illusion du mythe ou la persuasion du discours (Untersteiner 1993 [1967] : 210). Ainsi toute expérience de connaissance en prise sur l'action est-elle vouée à l'échec, à moins de changement de thématique, c'est-à-dire en renversant l'advenu d'un contenu et d'un mode de pensée en son contraire. Passage de l'illusion à la persuasion ou vice versa - la persuasion démasquée en leurre -, qui ne peut se faire qu'au moment opportun choisi à dessein par l'orateur ou le dramaturge. On ne change pas sans laisser derrière soi des préjugés et un mode de penser. Cet entre-deux en creux, que les Grecs appellent kairos, est le temps par excellence de l'acte. C'est le moment favorable et adéquat, non prédéterminé, qui, en fonction d'une situation donnée et de ses paramètres fait coupure et ouverture (Cassin 2012 : 166). En dénonçant un défaut de cuirasse dans une argumentation et en y substituant une autre, Gorgias amorce un changement de discours

5. Cf. Platon, «Protagoras ou les sophistes» (in 1950: 73-146).

6. Ibid. : 323. 
et un changement d'état. Il a pu ainsi soutenir qu'il fait avec les mots ce que le médecin fait avec le remède: "De même que les drogues", dit-il, "tirent des dispositions différentes au corps, que certaines mettent fin à la maladie, d'autres à la vie, de même les mots peuvent provoquer la joie ou le chagrin, la peur ou la confiance " (Éloge d'Hélène, 14). Parole qui libère, parole qui soumet. Ainsi le langage est-il pour les sophistes non pas un organon, "un instrument maîtrisable " à la manière d'Aristote, mais un pharmacon, un moyen de soigner ou d'empoisonner (Cassin 2012 : 93).

En somme, en appliquant la logique binaire vrai/faux et le principe de non-contradiction qui en découle, le discours apodictique des philosophes subordonne la parole à une sémantique unilatérale qui vise, au sein de la réalité, une unité universellement stable - l'être de l'étant. En revanche, le discours épidictique des sophistes joue en permanence sur l'ambiguïté des idées et des sensations, et sur les antinomies des drames humains afin de faire, dans une situation particulière donnée, basculer en un temps jugé favorable (kairos) une opinion unanimement admise en une opinion nouvelle dont la véracité est évaluée après coup dans ses effets ${ }^{7}$.

Les logiques discursives apodictique et épidictique, qui se sont mises en place dans l'Antiquité en se récusant mutuellement, nous intéressent car les croyances instituées dans les sociétés traditionnelles ont pour principale caractéristique - on l'oublie trop souvent - d'être épidictiques. Que ce soit dans la magie, la religion ou la sorcellerie, l'efficience d'une formule rituellement proférée, d'une offrande sacrificielle ou d'un jugement par ignorance se vérifie dans l'après-coup de leur effectuation, laquelle est cautionnée par des instances tierces. Si donc le savoir et la connaissance apodictiques dépendent de la logique binaire vrai/faux, $\mathrm{bien/mal,} \mathrm{qui} \mathrm{est} \mathrm{au} \mathrm{fondement} \mathrm{de} \mathrm{leur} \mathrm{argumentation} \mathrm{et} \mathrm{de} \mathrm{leur}$ développement, les croyances épidictiques répondent à une logique ternaire selon laquelle les relations entre les sujets sont pré-ordonnées par l'altérité tierce dont elles relèvent : puissances tutélaires de la religion, objet impersonnel et supposé efficient de la magie, au-delà bivalent de la sorcellerie. Il en va de même pour le discours qui sous-tend les alliances matrimoniales, à ceci près que la sexualité sur laquelle il se fonde relève d'une altérité qui dénote par une absence et qu'éclaire la théorie de Lacan.

\section{L'alliance matrimoniale entre dette symbolique et échange}

Selon cette approche psychanalytique, le désir s'oppose en premier lieu au besoin, même si, dans la vie quotidienne, ces deux fonctions se recouvrent continûment. Tandis que le besoin, déterminé par ses fins, est centré sur

7. L'Éloge d'Hélène de Gorgias est, à cet égard, la parfaite illustration de ce type de discours. 
sa satisfaction qui assure la régulation et la survie physiologiques du corps, le désir, générateur de la sexualité, procède originellement dans l'inconscient d'un manque qui soutient sa vitalité tant qu'il n'est pas comblé. C'est dans ce manque que viennent se loger, tour à tour ou dans le désordre, les objets moteurs - oral, anal, scopique, phallique - des pulsions, qui, par le biais des représentations érotisées du corps de l'autre qu'elles impulsent, sous-tendent le rapport du sujet à la jouissance. Le désir et la jouissance constituent ainsi les deux principaux pôles de la sexualité qui, bien qu'interdépendants - l'un n'existant pas sans l'autre -, sont foncièrement antagonistes. Si le désir fait exister la jouissance et que celle-ci donne corps au désir, on sait aussi qu'un excès de jouissance tue le désir lorsqu'il sature son manque, et que le désir n'a de cesse d'endiguer les débordements de la jouissance selon certaines modalités - inhibition, symptôme, angoisse - que Freud a décryptées en pionnier (1993 [1926]). Le désir et la jouissance peuvent ainsi se manifester sous des formes extrêmes. D'un côté, par son absence d'objet initial, le désir est au principe de la pudeur, laquelle cache plus la fragilité de son manque interne que la visibilité de ses organes externes (Lacan 1994: 166). Ce manque, qui est protégé par les règles de la convenance dans notre société, et aussi par des rites de séparation dans les sociétés traditionnelles, a pour vertu d'établir entre les sujets une distance passive et neutre posée en tiers. Dans la sexualité, on ne montre pas tout et on ne dit pas tout sinon à sombrer dans l'indécence destructrice du désir. D’un autre côté, lorsque la jouissance est en proie aux impératifs des pulsions, elle exerce une tension entre moi et l'autre, et une aliénation au corps fantasmé de l'autre qui peuvent être l'objet d'attraction et de répulsion aussi bien conjointes que disjointes. La jouissance est ambivalente par nature. La libération de ses tensions s'effectue communément par le biais de la "petite mort " propre à l'orgasme qui précipite le retour de la jouissance au "point mort» du désir. Mais, à défaut de cette sortie usuelle, la jouissance peut être source de conflits sans fin attisés par la jalousie, la haine ou les sentiments de persécution. En bref, autant le désir est séparateur et pondérateur, donc pacifique, autant la jouissance, fusionnelle et passionnelle, est conflictuelle.

Cette double polarité de la sexualité est au cœur du développement de l'alliance matrimoniale des Bangoua qui se déroule en trois temps définis par trois types de redevances, c'est-à-dire par trois rapports à l'autre. Lorsque les fiançailles sont officiellement scellées, le fiancé est invité à donner de nombreux présents aux personnalités importantes du lignage de sa future et à verser à son beau-père une somme d'argent en espèces, dont le montant est surévalué par rapport à ses possibilités financières et à celles des membres de sa famille qui l'aident à payer. Dans le premier 
temps de cette transaction, le fiancé donne ce qu'il peut aux uns et aux autres, mais, quoi qu'il donne, il est toujours redevable, la dette contractée étant incommensurable. La valeur d'échange offerte n'opérant pas une cession, la fille n'est pas séparée de son père et de son lignage. C'est pourquoi, dans un deuxième temps, il est stipulé que l'alliance doit être scellée en dernière instance par un tiers, le grand-père maternel de la fiancée, lequel, n'appartenant pas dans cette société patrilinéaire au lignage de sa petite-fille, réclame pour la cession de celle-ci un prix ordinaire, une chèvre et une calebasse d'huile de palme, que tout homme peut facilement régler. Cette valeur d'échange banalisée est alors soldée non pas par l'acquisition d'une valeur d'usage qui, selon la logique des échanges marchands, aliénerait la femme échangée à son détenteur, mais par une dette symbolique qui la sépare des siens sans pour autant l'aliéner à son mari, puisque la dette est sans contenu. Acquérir une femme n'équivaut pas dans ce contexte à un crédit, mais à une dette. Enfin, dans un troisième temps inauguré par l'entrée de la femme dans la demeure maritale, le partage des tâches et les échanges réciproques qui président à la vie quotidienne prennent le dessus. Les époux travaillent tous les deux et font chacun de leur côté du commerce, dont le bénéfice est, pour une part, destiné à la vie commune et, pour l'autre, à des fins personnelles. Les femmes bamiléké, à l'instar des femmes africaines en général, sont indépendantes socialement et économiquement.

Les trois temps de cette alliance matrimoniale, qui s'articulent sur un jeu de redevances, dette infinie, dette symbolique et dette de réciprocité - c'est-à-dire sur trois rapports à l'autre : conflit, séparation symbolique et intégration dans la vie quotidienne -, relèvent non pas d'une économie marchande, c'est patent, mais d'une logique sociale fondée sur les lois de la subjectivité et de la sexualité. Dans un premier temps, la fiancée représente pour les donneurs et le preneur de femmes une valeur de jouissance, dont la valeur d'échange est communément exploitée par les prostituées qui en font leur commerce. Cependant ici, cette valeur de jouissance fait surtout l'objet d'une surenchère, laquelle, poussée à son paroxysme par le prix surévalué exigé par le père de la fiancée, ne fait qu'exacerber les contradictions qui lui sont inhérentes. La jouissance, ambivalente et conflictuelle par essence, ne peut séparer la valeur attribuée par le père à sa fille de celle de l'épouse que doit recevoir le fiancé. Quoi que donne le fiancé, il ne reçoit rien, car la cession de la fille pour devenir épouse et mère est impossible. Pour sortir de cette impasse, source de frustration pour les fiancés, l'intervention du grand-père maternel de celle-ci est, dans un deuxième temps, décisive, car, en tant qu'allié du lignage de la fiancée appelé à sceller officiellement l'alliance matrimoniale de la fille de sa fille, 
il reconduit la dette symbolique en chaîne, qui, transmissible d'une génération d'alliés à une autre, fait acte à chaque nouvelle alliance au sens que nous avons attribué à ce terme. Cette dette inaugure pour les époux leur relation conjugale qui repose, non pas comme dans notre civilisation sur une union à construire, mais sur une séparation à reconduire : séparation pacifique entre lignages alliés, d'une part, et entre époux, d'autre part, qui se doivent mutuellement du respect à partir du moment où ils partagent une intimité commune. La dette symbolique crée ensuite une irréversibilité par rapport à la dette infinie contractée au départ par le fiancé - cet état conflictuel initial est dépassé -, et par rapport à l'origine familiale de la fiancée - sa descendance appartiendra au lignage de son mari. Enfin, la dette symbolique, qui reconduit le manque du désir d'une génération d'alliés à l'autre, est rétroactive car ses effets se réalisent pour les époux et leurs descendants dans l'après-coup de son instauration sous les formes conjointes d'une libération subjective et d'une résolution de conflit. Dans cette transaction matrimoniale actée, les femmes ne sont en rien des objets inaliénables - des valeurs d'usage -, mais des sujets aliénables qui, en participant d'un manque, sont "Pas-toutes ", c'est-à-dire des sujets désirants protégés par la pudeur. Enfin, lors du troisième temps de l'alliance matrimoniale, le partage des tâches quotidiennes, fondées sur les identifications traditionnelles aux aînés et les échanges régulés par le jeu de la réciprocité, reprend son cours. La dette symbolique tombe dans l'oubli, on n'en parle plus, sinon lorsqu'elle fait défaut lors des conflits et des maladies. La thérapie locale consistait justement à la rétablir.

Il y a encore une trentaine d'années, le guérisseur, appelé nggangkang, "celui qui possède un pouvoir, $k a n g$, lié à la forêt », recevait des patients affectés par une maladie physique, un malaise psychologique ou une infortune, qui pensaient unanimement que leur malheur était dû à un sorcier ou à une sorcière. Persécutés et frustrés, ils espéraient que le guérisseur, doté d'un pouvoir exceptionnel venant de la forêt, les désensorcellerait. Le nggangkang les écoutait faire part de leurs maux et consultait ensuite l'araignée mygale, l'instance divinatoire des Bamiléké. Cet augure lui indiquait le plus souvent de demander aux patients d'apporter des offrandes de nourriture aux ancêtres de leur grand-père maternel, auxquels leur père est redevable d'une dette symbolique contractée initialement lors de son alliance matrimoniale. Il s’agit ainsi de les amener à se réinscrire dans un manque que leur désarroi psychologique a momentanément effacé. Cette thérapie traditionnelle, qui instaure un non-retour à l'état antérieur de la sorcellerie et inaugure un nouvel état subjectif pacifié, est parallèle aux étapes par lesquelles je suis passé dans les premiers temps de mon séjour à Bangoua. C'est après avoir souffert les affres d'une affaire 
de sorcellerie que j'ai mesuré ensuite, en tant que Tiénja' - en tant que petit-fils allié du chef -, à quel point la relation d'alliance ancrée dans la dette symbolique peut être source de libération et ouverte à la plaisanterie. C'est dire que les effets dans l'après-coup de l'acte thérapeutique, qui sont particuliers pour chaque sujet, ne peuvent être évalués objectivement et statistiquement. La guérison est sensible mais pas mesurable. De toute manière les patients en témoignent toujours discrètement. Enfin, dans le troisième temps de ce traitement, le guérisseur demandait aux patients de revenir le voir en apportant, selon leur sexe, un coq ou une poule avec lesquels il essuyait leur corps afin de chasser définitivement le mal dont ils souffraient. "Maintenant, la mauvaise chose est finie", disait-il pour conclure la cure. Le patient était alors invité à se réinsérer dans le jeu des partages et des échanges de la vie quotidienne d'une façon différente.

Les trois temps rituels qui ponctuent les transactions matrimoniales et les thérapies sont canoniques - bien qu'ils n'aient jamais été considérés jusqu'à présent comme tels, à l'instar des trois temps des rites de passage d'Arnold Van Gennep (1969 [1902]) -, car ils sous-tendent de nombreux rites dans d'autres sociétés, tels que, pour ne citer que deux exemples éloignés l'un de l'autre, l'initiation encore actuelle des jeunes garçons pèrè du Cameroun (Pradelles de Latour 2005: 93-106) et celle des filles recueillie par Margaret Mead chez les Arapesh de Papouasie-NouvelleGuinée avant la Seconde Guerre mondiale (1963 [1935] : 79-85). Avec leurs trois phases préétablies, les rites constituent un espace-temps protégé des aléas de la vie quotidienne, où l'acte propre à l'ordre du désir peut être scellé et créer une séparation dont les effets rétroactifs sont donnés dans un après-coup particulier pour chaque sujet. Ainsi le rite institué en trois temps est-il un paradigme de l'acte, lequel ne doit rien aux modalités de l'action. C'est l'acte qui fait qu'un rite thérapeutique est homologue à un rite de passage, et réciproquement tout rite de passage qui fait acte est thérapeutique.

Cette conception de l'alliance matrimoniale ancrée dans la dette symbolique séparatrice s'oppose-t-elle à la théorie de Claude Lévi-Strauss fondée sur les échanges de femmes? La question mérite d'être posée car ces deux approches de l'alliance matrimoniale sont apparemment antithétiques, mais le sont-elles vraiment? Si on se réfere à l'ethnographie des Baruya de Papouasie-Nouvelle-Guinée, Maurice Godelier explique que dans cette société patrilinéaire où les hommes échangeaient leurs sœurs, les deux beaux-frères ne se tiennent pas quittes pour autant de toute obligation. Bien au contraire, redevables mutuellement d'une dette d'alliance non comptable, non réciproque, celle-ci renforce leur coopération. Les beaux-frères multiplient les échanges de services et de cadeaux, et en 
viennent à compter l'un sur l'autre, bien plus que sur l'aide de leurs propres frères (Godelier 2003 [1982] : 51). Chez les Bamiléké aussi, qui relèvent comme les Samo de Françoise Héritier des structures semicomplexes de l'alliance matrimoniale, l'échange des sœurs était pratiqué à condition que ce redoublement d'alliance ne soit réitéré que toutes les trois générations et dans des lignées masculines différentes (1981: 112-113). Si ces deux formes d'alliance matrimoniale coexistent dans ces deux sociétés, on peut gager qu'elles existent aussi dans bien d'autres. D'où la question, comment cette concomitance est-elle possible? C'est parce qu'elles relèvent de deux discours opposés mais complémentaires.

Les structures de la parenté lévi-straussiennes, qui reposent sur différentes modalités de l'échange - restreinte, généralisée, discontinue, semi-complexe, complexe - définies par un jeu d'oppositions hommes/ femmes, consanguins/alliés, aînés/cadets, de type binaire à la fois mentales et sociales, ont pour fonction de maintenir une cohésion d'ensemble dans les esprits et entre les groupes de filiation ou les classes matrimoniales d'une société afin d'assurer leur reconduction. Ce sont des systèmes téléologiques. Les rapports ordonnés entre les sexes et les générations qui ont pour but leur reproduction sont construits par un savoir semiconscient que les donneurs et les receveurs de femmes appliquent au mieux en fonction des réalités. Les hommes y jouent un rôle dominant comme le soutient Claude Lévi-Strauss (1967 [1948]: 134-136), et le reprend Françoise Héritier (2002) pour définir l'opposition entre les deux sexes. La conception de l'alliance fondée sur les échanges de femmes tient ainsi sa pertinence du discours apodictique mis en ouvre par des sujet-agents maîtres de leur action. Mais la sexualité qui sous-tend les transactions matrimoniales ne doit rien à la reproduction humaine et dépend non pas directement de rapports sociaux établis, mais d'une instance tierce dont la dette symbolique d'alliance dénote l'altérité. "Quand il s'agit de sexe", dit Lacan, "il s'agit toujours de l'Autre sexe même quand il se réfere au même ${ }^{8}$. Dans cette position subjective les deux sexes sont à la même enseigne, l'un ne domine pas l'autre car la sexualité est coupure et rétroactive pour les deux partenaires. L'effet libérateur qui se manifeste dans l'après-coup de sa réalisation est à la base des discours épidictiques et des coupures opérées lors des rites de passage. Celles-ci ne servent pas seulement à ponctuer les étapes d'un cycle de vie - naissance, initiation, mariage, mort -, mais aussi à assurer le renouvellement du désir pour les sujets. Autrement dit, les discours épidictique et apodictique divisent non seulement les sujets-effets des sujets-agents,

8. Jacques Lacan, Le Savoir du psychanalyste, séminaire inédit du 4 mai 1972. 
mais aussi les sociétés traditionnelles selon deux types de pratiques: les séparations rituelles et les relations usuelles. Dès lors, il n’y a plus lieu d'opposer la subjectivité à la société comme on le fait traditionnellement en anthropologie (Godelier 2013 [1982] : 15), mais de montrer que ces domaines sont tous deux traversés par la même division. De même qu'il y a deux conceptions du sujet, il y a deux modalités du social, lesquelles sont plus marquées dans les sociétés traditionnelles que dans la nôtre parce que le manque y est partiellement institué sous la forme d'une dette symbolique. Ainsi, à l'instar des deux visages de Janus, l'alliance matrimoniale d'un grand nombre de ces sociétés regarde dans deux directions opposées tout en appartenant à la même tête. D'un côté, une forme institutionnelle apodictique socialement établie dans les esprits, de l'autre, une absence de contenu épidictique, la source des actes manqués - réussis en tant que coupures - d'où procèdent le désir et la vie.

\section{Centre national de la recherche scientifique} ch.pradelles@dartybox.com

MOTS CLÉS/KEYWORDS: Bamiléké - alliance matrimoniale/matrimonial alliance - échange matrimonial/matrimonial exchange - dette symbolique/symbolic debt - discours épidictique/ epideictic discourse - discours apodictiquelapodictic discourse - sophistes/sophists - Éléates/ Eleatics - jouissance - désir/desire. 


\section{RÉFÉRENCES CITÉES}

Austin, John Langshaw

1970 Quand dire c'est faire.

Trad. par Gilles Lane. Paris, Le Seuil

("L'ordre philosophique»).

\section{Cassin, Barbara}

1986 «Du faux ou du mensonge à la fiction (De pseudos à plasma)", in Barbara Cassin, ed., Le Plaisir de parler. Études de sophistique comparée.

Paris, Minuit ("Arguments») : 3-29.

2012 Jacques le sophiste. Lacan, logos et psychanalyse. Paris, EPEL («Essais»).

\section{Ducrot, Oswald}

1972 Dire et ne pas dire.

Principes de sémantique linguistique.

Paris, Hermann («Savoir »).

Freud, Sigmund

1993 [1926] Inhibition, symptôme et angoisse. Trad. de l'allemand par Joël et Roland Doron. Paris, Presses universitaires de France ("Quadrige» 172).

Godelier, Maurice

2003 [1982] La Production des grands hommes. Pouvoir et domination masculine chez les Baruya de Nouvelle-Guinée. Paris, Flammarion ("Champs» 540).

2013 Lévi-Strauss. Paris, Le Seuil.

Guthrie, William Keith Chambers

1976 Les Sophistes. Trad. de l'anglais par Jean-Pierre Cottereau. Paris, Payot ("Bibliothèque historique").

\section{Héritier, Françoise}

1981 L'Exercice de la parenté. Paris, Gallimard-Le Seuil ("Hautes études»). 2002 Masculin-féminin II. Dissoudre la hiérarchie. Paris, Odile Jacob.

Lacan, Jacques

1994 Le Séminaire, 4. La relation d'objet. Paris, Le Seuil (« Le Champ freudien»).
Lévi-Strauss, Claude

1967 [1948] Les Structures élémentaires de la parenté. Paris-La Haye, Mouton.

\section{Mead, Margaret}

1963 [1935] Mours et sexualité en Océanie. Trad. de l'américain par Georges Chevassus. Paris, Plon («Terre humaine»).

\section{Parménide}

1955 Le Poème de Parménide.

Éd. par Jean Beaufret. Paris, Presses universitaires de France («Épiméthée » 11).

\section{Platon}

1950 Euvres complètes. Trad. et éd. par Léon Robin. Paris, Gallimard («La Pléiade» 58).

\section{Pradelles de Latour, Charles-Henry}

1997 Le crâne qui parle. Paris, EPEL [1 $1^{\mathrm{re}}$ éd. : Ethnopsychanalyse en pays bamiléké. Paris, EPEL, 1991].

2005 Rites thérapeutiques dans une société matrilinéaire. Le gèrem des Pèrè (Cameroun). Paris, Karthala ("Hommes et sociétés»).

2014 La Dette symbolique.

Thérapies traditionnelles et psychanalyse.

Paris, EPEL («Essais»).

2015 «La dette symbolique d'alliance matrimoniale et sa fonction thérapeutique ", Cultures-Kairós

[http ://revues.mshparisnord.org/cultureskairos/index.php ?id=1196].

\section{Untersteiner, Mario}

1993 [1967] Les Sophistes. Trad. de l'italien et éd. par Alonso Tordesillas. Paris, Vrin («Bibliothèque d'histoire de la philosophie»).

\section{Van Gennep, Arnold}

1969 [1902] Les Rites de passage. Étude systématique des rites de la porte et du seuil, de l'hospitalité, de l'adoption, de la grossesse et de l'accouchement. Paris-La Haye, Mouton. 
Charles-Henry Pradelles de Latour, L'alliance matrimoniale des sociétés traditionnelles: entre discours épidictique et discours apodictique, entre dette symbolique et échange. - L'auteur raconte comment, sur son premier terrain africain en pays bamiléké, sa neutralité dans une affaire de sorcellerie lui valut d'être appelé Tiènja', un nom réservé aux fils de filles du chef, lui conférant un statut d'allié du chef, auquel il était désormais lié par une dette symbolique d'alliance matrimoniale.

Cette dette d'alliance introduit un ordre de discours totalement différent du discours courant. D'une part, elle relève du discours épidictique où son absence de contenu est reconnue dans l'après-coup de son effectuation et non avant comme dans le discours apodictique. D'autre part, à la différence de l'action qui vise une fin, la dette symbolique fait acte, en instaurant une séparation inaugurale spécifique des relations d'alliance matrimoniales. Enfin, l'absence de contenu de la dette symbolique sous-tend la relation du sujet au désir qui le tient à l'écart des équivoques, des contradictions et des tensions intrinsèques au jeu de la jouissance, dont, entre autres, se nourrissent les affaires de sorcellerie.
Charles-Henry Pradelles de Latour, The Matrimonial Alliance in Traditional Societies: Between Epideictic and Apodictic Discourses, Between Symbolic Debt and Exchange. - The author tells how, on his first African fieldwork in the Bamileke country, his neutrality during accusations of witchcraft earned him to be called Tienja', a name which is traditionally given to the chief's daughter's sons. To receive such a name means to be related to the chief by a relation of matrimonial alliance based on a symbolic debt.

The symbolic debt introduces an order of discourse which is quite different from the order of the daily discourse. On the one hand, the symbolic debt belongs to the epideictic discourse which implies that its absence of meaning is recognised after having been played, and not before as it is usual in the apodictic discourse. On the other hand the symbolic debt certifies an inaugural separation between the lineages which is specific to the order of the matrimonial alliance. Last but not least, the lack of sense of the symbolic debt underlies the relation of the subject to the desire, and it helps him to steer clear of equivocations, contradictions and states of nervous tension between the subjects, which are specific among others to the witchcraft accusations. 